\title{
Immunogenicity Evaluation of a Novel Lentiviral Vaccine Expressing Multi- Epitope Against of Leishmania Major in BALB/c Mice
}

\section{Mahsa Rabienia}

Fasa University of Medical Science

\section{Zahra Roudbari}

University of Jiroft

\section{Ali Ghanbariasad}

Fasa University of Medical Science

Abbas Abdollahi

Fasa University of Medical Science

Alireaza Molazadeh

Fasa University of Medical Science

Nahid Mortazavidehkordi

Fasa University of Medical Science

Akbar Farjadfar ( $\nabla$ farjadbio@gmail.com )

Fasa University of Medical Science

\section{Research}

Keywords: Cutaneous Leishmaniasis, Leishmania major, lentivirus, Vaccine, Th1 response, Th2 response

Posted Date: December 7th, 2020

DOl: https://doi.org/10.21203/rs.3.rs-72175/v1

License: (c) (i) This work is licensed under a Creative Commons Attribution 4.0 International License. Read Full License 


\section{Abstract}

Background: Nowadays, the prevention of parasitic diseases including leishmaniasis is one of the health concerns in the world, and cutaneous leishmaniasis is the most common type of these diseases. So far, no drug or vaccine has been approved for definitive treatment of this disease.

Methods: In this study, the recombinant lentiviral vaccine containing a new multi-epitope of KMP11 and HASPB of the Leishmania major (L. major) was synthesized that had previously been designed in-silico. The designed multi-epitope was subcloned into the pCDH513 lentiviral vector, and the recombinant lentiviral multi-epitope vaccine (rLV-multi-epitope) was synthesized in the HEK293T cell by the packaging vectors. Also, the Western Blotting method was used to confirm the gene expression. Then, the rLV-multiepitope vaccine was injected twice, along with two control groups, PBS, and rLV-empty to immunize the BALB /c mice. Twenty-one days after the second injection, the splenocytes of the mice were isolated and stimulated with the Leishmania lysate.

Results: The results of the enzyme-linked immunoassay (ELISA) test not only showed the titer of IFN- $\gamma$ and IL-4 was increased in the immunized group compared to the controls, but also indicated that the ratio of IFN-y to IL-4 cytokines in the main group was increased significantly. As a result, the Th1 response was generated in the main group. Moreover, the humoral immune response was assessed and the results showed that the ratio of $\lg$ G2a to $\lg \mathrm{G} 1$ antibody in the sera of the immunized mice was increased compared to the control groups. Also, the ratio of IgG2a to $\operatorname{lgG} 1$ was increased in the main group. Therefore, the humoral immune response was increased, which can also have a positive effect on increasing the Th1 response.

Conclusions: Our results showed that immunization by the new rLV-multi-epitope vaccine could stimulate the immune system towards the Th1 through increasing the IFN-y production.

\section{Background}

The outbreak of leishmaniasis is among the concerning health issues in many parts of the world, and it is estimated that 350 million people are at the risk for affecting with it around the world. Cutaneous leishmaniasis has the highest prevalence among the most common forms of the disease, with Leishmania major (L. major) as one of the causing factors (1).

Thus, there is a need for definite treatment of the cutaneous leishmaniasis due to the complications of this disease including the skin ulcers and social harms (2). Unfortunately, there is still a challenge for treatment, the drugs used have high costs, and side effects of these drugs may endanger one's health. Therefore, it is essential to design and develop a vaccine that can stimulate the immune system to provide the defense against the parasites (3).

Although, various types of vaccines including live -attenuated, killed parasites, subunit vaccines, recombinant proteins, and DNA vaccines have been studied in -vitro for treatment of the leishmaniasis in 
the past decade, but because of the contradictory observed results, the researchers have not yet reached a definitive conclusion on the use of these vaccines for treatment of the humans, and a few vaccines have entered the clinical trials $(1,4)$. Thus, there is an urgent need for development of a vaccine that can provide more effective immunity than the previous vaccines (5).

Among the types of vaccines studied, the use of DNA vaccines containing the clusters of differentiation (CDs) of whole immunogenic protein has shown promising results. But the use of these antigens in the form of proteins is accompanied with some problems, such as high production cost, short half-life, low immunogenicity, low stimulation of cellular immunity, and cross-allergic reactions (6). Therefore, in the synthesis of vaccines, the use of immunoinformatics tools can be very useful in order to increase the immunogenicity and decrease the adverse reactions (7).

The most appropriate multi-epitope vaccine can be designed to stimulate the immune system using the immunoinformatic servers and performing tests, such as T-cell prediction, docking, and molecular dynamic simulation (8). DNA vaccines expressing the multi-epitope have many advantages over DNA vaccines expressing a whole protein including: (i) they have several epitopes that can stimulate the helper T lymphocyte (HTL), cytotoxic T lymphocyte (CTL), and B cell lymphocytes and thus, can both develop cellular and humoral immune responses, (ii) in their design, several epitopes of different antigens can be used that can greatly stimulate the immune system; and (iii) they will prevent the unwanted reactions due to the allergen tests and other evaluations carried out on them in -silico $(9,10)$.

The most important step in the design of a multi-epitope vaccine is selecting the appropriate antigen to predict the epitopes. If the appropriate antigen is selected, one can hope to produce an effective vaccine (11). A definite approach to select the strong and effective antigens is predicting the antigenic epitopes proven in the previous studies to have an immunogenic effect, but it does not have the potential to become an effective vaccine due to the mentioned reasons (12).

Also, adding a linker can be very helpful for enhancing the immunogenicity of the vaccines. Among the linkers used, glycine-rich linkers that also contain the hydrophilic amino acids can provide high flexibility and bind the epitopes together in a construct without altering the performance of the domains (13).

In our previous studies, we found that the lentiviral vaccine containing the kinetoplastid membrane protein 11 (KMP11) and hydrophilic acylated surface protein B (HASPB) whole proteins are capable of stimulating the immune system in the BALB/c mice (14). Therefore, in this study, the immunogenic effects of the epitopes of these two antigens were investigated.

In the present study, the lentiviral vector with the GDGDG linker and HTL and CTL antigen epitopes selected by the immunoinformatics server was introduced to increase the immunogenicity and efficacy of the vaccine. After producing the recombinant lentiviral multi-epitope, the immunogenicity of this vaccine was evaluated in the BALB/c mice.

\section{Methods}




\section{Design of multi-epitope lentiviral vaccine}

The protein sequences of KMP11 and HASPB of L.major (Friedlin strain) were obtained from GenBank, protein database of the National Center for biotechnology information (NCBI, http://www.ncbi.nih.gov/genbank/). The ID of KMP11 and HASPB was AAR84616.1 and CAB39972.1, respectively. Then, with the help of immunoinformatics software such as T- cell epitope prediction, B-cell epitope prediction, docking studies and molecular dynamics simulation, the epitopes of KMP11 and HASPB proteins were selected in silico space (15). Also, using GDGDG linker, final multi-epitope vaccine was designed (Figure. 1).

\section{Cell culture}

HEK293T cell line (Human Embryonic Kidney, ATCC CRL-3216) was purchased from Mede Bioeconomy Company Iran and seeded in Dulbecco's modified Eagle's medium (DMEM; Gibco, Darmstadt, Germany). The cells were supplemented with $10 \%$ foetal bovine serum (FBS) (Gibco, USA), penicillin (100 units $/ \mathrm{ml}) /$ streptomycin $(100 \mathrm{mg} / \mathrm{ml}$ ) (Invitrogen, Carlsbad, CA, USA), finally the cells were incubated in a humidified atmosphere with $5 \% \mathrm{CO} 2$ at $37^{\circ} \mathrm{C}$.

\section{Synthesis and confirmation of the pCDH-multi-epitope construct}

The designed multi-epitope was synthesized by Genscript, USA, and merged into pUC57 plasmid. Then, the construct was subcloned into pCDH513B lentiviral vector using Xbal and $\mathrm{BamHI}$ restriction enzymes. The recombinant $\mathrm{pCDH}$-multi-epitope was digested using $\mathrm{Xbal}$ and $\mathrm{Apal}$ and sequenced. In this construct, to express multi-epitope cytomegalovirus (CMV) promoter was used. Also, GFP and puromycine were expressed using eukaryotic elongation factor (eEF) promoter. Moreover, GFP and puromycine were separated by T2A factor. As a result, transfer vector expressed multi-epitope, GFP and puromycine resistance factor from two mRNAs (Figure. 2).

The pCDH-multi-epitope plasmid was used to transform the competent E. coli DH5a cells. After transformation of bacteria, the LB agar containing ampicillin $(100 \mu \mathrm{g} / \mathrm{ml})$ was used to bacteria culture at $37^{\circ} \mathrm{C}$ for $24 \mathrm{~h}$. Then, from the colonies appeared on the plate, plasmid extraction was carried out using Miniprep plasmid extraction kit (Qiagen, Germany) according to manufacturer's protocol. Afterward, the restriction enzymes containing $\mathrm{Xbal}$ and $\mathrm{Apal}$ were used to digest the extracted plasmid and electrophoresed on $1 \%$ agarose gel. Finally, sequencing of plasmid was done to further confirm the cloning. Also, in order to extract of PAX, MD, and pCDH lentiviral plasmids, all procedures were performed.

\section{Virus production (rLV-multi-epitope and rLV-empty)}

By using the transfer lentiviral vector ( $\mathrm{pCDH}$-multi-epitope or pCDH-empty) and two PAX and MD plasmids, transfection of HEK293T cells were done based on Trono protocol (calcium phosphate transfection method)(16). On the first day, $3 \times 10^{6}$ HEK293T cells were seeded in a $10-\mathrm{cm}$ plate in Dulbecco's modified Eagle's medium (DMEM) (Gibco, USA), supplemented with 10\% FBS (Gibco, USA). On 
the second day, a mixture of $21 \mu \mathrm{g}$ of the transfer vector (pCDH-multi-epitope or pCDH-empty), $21 \mu \mathrm{g}$ of psPAX2 vector and $10 \mu \mathrm{g}$ of pMD2.G vector with transfection buffer was added dropwise to the cells. Then, 14 hours' post-transfection, fresh medium was used to replace the previous medium. After 24 hours, with the help of a fluorescent microscope, evaluation of GFP expression was performed. Moreover, 24, 48 and 72 hours' post-transfection the supernatant of the packaged lentivirus (named thereafter rLVmulti-epitope or rLV-empty) were harvested and centrifugation at $350 \mathrm{~g}$ at $4^{\circ} \mathrm{C}$ for 5 minute was done. Moreover, the supernatant was filtered through a $0.22-\mu \mathrm{m}$ filter. Finally, the rLV-multi-epitope or rLV-empty were stored at $-70^{\circ} \mathrm{C}$ for next experiments.

\section{Titarion of virus}

Using flow cytometry, the number of GFP-positive cells were counted and, the titre of packaged viruses was defined. See the virus count formula

$\mathrm{TU} / \mathrm{mL}=(1 / 4)(F * N * D * 1000) / V$, Where $F=$ percentage of fluorescent cells (EGFP), $N=$ number of cells at the time of transduction (about $1 \times 10^{5}$ cells per well), $D=$ fold dilution of vector sample used for transduction and $V=$ volume $(\mathrm{mL})$ of diluted vector sample added into each well for transduction. All steps have been previously described(17).

\section{Western blot}

In order to confirm the expression of rLV-multi-epitope, western blot was carried out (18).

The First, the rLV-multi-epitope and rLV-empty were used to transduce of HEK293T cells. Thereafter, the cells were treated with $2 \mu \mathrm{g} / \mathrm{mL}$ puromycin (Sigma-Aldrich, USA). Subsequently, the transduced HEK293T cells (rHEK-multi-epitope, rHEK-empy) and non-transduced HEK293T cells (HEK-non) were seeded in 6well plates. Then, after $72 \mathrm{~h}$, the cells were trypsinized and centrifuged at $190 \mathrm{~g}$ for $5 \mathrm{~min}$ at $4{ }^{\circ} \mathrm{C}$. Also, the cells were lysed in $200 \mu \mathrm{L}$ of protease inhibitor-containing RIPA buffer (Thermo Fisher Scientific, IL). The lysates were centrifuged at $8800 \mathrm{~g}$ for $12 \mathrm{~min}$ at $4{ }^{\circ} \mathrm{C}$. Then, the protein concentration of the supernatants was determined using a BCA Protein Assay Kit (Thermo Fisher Scientific, IL). Lysates/lane of $30 \mu \mathrm{g}$ were loaded onto a 12\% SDS-PAGE gel and then transferred onto a nitrocellulose membrane (Bio-Rad Laboratories, CA).

Thereafter, the protein was transferred onto a nitrocellulose membrane (Bio-Rad Laboratories, CA). Then, using 2.5\% non-fat milk, the membranes were blocked and using Anti-polyHistidine antibody (anti-His antibody) (Abcam, USA) conjugated with horseradish peroxidase (HRP) at 1:2000 dilution immunoblotting was performed.

Finally, to visualize the protein band, the substrate of the tetramethylbenzidine (TMB) was used. Finally, the optical density (IOD) of the band was obtained and it was integrated.

\section{Immunization of BALB/c mice}


Experiments were performed on twenty-four female inbred BALB/c mice, 6-week-old which were purchased from Pasteur Institute of Iran and were maintained in the Institutional animal care of the Fasa University of medical sciences based on the specific National Ethical Guidelines for Biomedical Research issued by the Research and Technology Deputy of Ministry of Health and Medicinal Education (MOHME) of Iran (2005).

Three groups of mice were considered and each group was randomly received 8 mice $(n=8)$, group 1 (rLV-multi-epitope), group 2 (rLV-empty) and group 3 (PBS). The injections were carried out subcutaneously twice during the 1 weeks (with a 3-day interval) with $1 \times 10^{6} \mathrm{TU}$ of rLV-multi-epitope $\left(3 \times 10^{6} \mathrm{TU} / \mathrm{mL}\right)$ to group 1 , and $1 \times 10^{6} \mathrm{TU}$ of rLV-empty $\left(1 \times 10^{7} \mathrm{TU} / \mathrm{mL}\right)$ and $0.1 \mathrm{~mL}$ PBS to control groups. Finally, three weeks post-immunization of mice, cytokine assay was done.

\section{Parasite culture}

In order to obtain a sufficient number of L. major (MHRO/IR/75/ER (parasite, promastigote parasites were cultured and enriched in RPMI 1640 (Gibco, UK) supplemented with 20\% foetal calf serum (Gibco, Darmstadt, Germany), $100 \mathrm{IU} / \mathrm{ml}$ of penicillin and $100 \mu \mathrm{g} / \mathrm{mL}$ of streptomycin. $100 \mathrm{IU} / \mathrm{mL}$ of penicillin and $100 \mu \mathrm{g} / \mathrm{mL}$ of streptomycin to obtain an adequate number of promastigotes, as described by Dehkordi et al (19).

\section{Antibody analysis}

To investigate the humoral immune response, three-week post-injection the sera of vaccinated mice were collected and analyzed in triplicate as previously described. To determine the presence of antibodies, 96well flat-bottom plates were coated with $L$. major promastigotes that there was obtained from stationary phase of culture. To block the plates, 1\% (w/v) BSA was used. Also, mice sera were diluted 1:100 and applied to the plate. Then, detection of attached antibodies was done, and the plate was incubated with anti-His antibody (Abcam, USA) and developed with peroxidase substrate tetramethylbenzidine (TMB). Finally, we used an ELISA microplate reader (Bio-Rad) to read the plate at $410 \mathrm{~nm}$.

\section{Cytokine analysis}

In order to evaluate the level of cytokine expression, the splenocytes of mice were separated and analyzed as previously complained. Three weeks after injection, in aseptic conditions the spleens of immunized mice and control groups were separated and homogenized. Then, the splenocytes were recovered, washed three times, and seeded in triplicate in a 96-well plate. Moreover, the cells were stimulated using L. major lysate, Soluble Leishmania Antigen (SLA).

Finally, to determine the levels of IL-4 and IFN- $y$, after 72 hours Supernatants were harvested and mentioned cytokines were assayed in triplicate by using sandwich ELISA kits (Abcam, USA) based on the manufacturer's protocol.

\section{Statistical analysis}


According to Mann-Whitney U-test, data analysis was performed and SPSS (V.18). A $P$-value $<0.05$ was considered as statistically significant.

\section{Results}

\section{Construction of rLV- multi-epitope vector}

Figure 1 shows the construct synthesized in this study. pUC57-multi-epitope was sub-cloned in pCDH513 lentiviral vector successfully. Using restriction enzymes Apal and $X b a /$ two fragments were obtained with Lengths of 1478 and $6355 \mathrm{bp}$ (Figure. 3). Furthermore, sequencing was performed, and as a result, the cloning of the construct was confirmed.

\section{Packaging And Titration Of The Recombinant Lentiviral Particles}

Using the fluorescent microscopy, evaluation of cGFP expression was performed and the packaging of the recombinant lentivirus verified. Using pCDH-multi-epitope, transfection of more than $90 \%$ of HEK $293 \mathrm{~T}$ cells after 72 hours were successful (Fig. 4a). According to expression of cGFP, the titre of the recombinant virus (rLV-multi-epitope) and empty vector (rLV-empty) was $1 \times 10^{6} \mathrm{TU} / \mathrm{mL}$, and $1 \times 10^{7}$ $\mathrm{TU} / \mathrm{mL}$, respectively.

\section{Transduction And Expression Of Rlv-multi-epitope}

The expression of rLV-multi-epitope at protein level was assessed and Western blot analyse was done. The expression of the multi-epitope in the trancduced HEK293T cell in the presence of a $10 \mathrm{KD}$ band was confirmed (Figure.3b). Transduced with the rLV-empty was used as a control and no bands was observed.

\section{Igg1 And Igg2a Assay}

In order to evaluate the ability of rLV-multi-epitope vaccine in the induction of humoral immune response, three post-injection, the sera from mice immunized with rLV-multi-epitope and the control groups were collected and titre of IgG1 and IgG2a antibodies against $L$. major was detected. According to ELISA test the rLV-multi-epitope vaccinated mice had a significantly higher titre of IgG2a and IgG1 compared to control groups (PBS and rLV-empty) $(P<0.05)$, and, the level of IgG2a was significantly $(p<0.05)$ higher than IgG1 level (Figure. 4a, b, and c).

\section{II-4 And Ifn-y Concentration}


The ability of vaccinated mice splenocytes to induce the Th1 or Th2 immune response was evaluated using Leishmania lysate or SLA. For this purpose, the level of IFN-y (Th1) and IL-4 (Th2) was measured in the supernatant of splenocytes of vaccinated mice with rLV-multi-epitope and control groups. As a result, the level of IFN-y and IL-4 produced by splenocytes of immunized mice were significantly higher as compared to control groups $(P<0.05)$. Also, an important finding is that IFN-y level was significantly $(P<$ 0.05 ) higher than that of IL-4 level in the main group compared to control groups (Figure. 5a, b, and c).

\section{Discussion}

In the recent years, a variety of vaccines have been investigated against different species of Leishmania parasite due to the ineffectiveness of the drugs used for treatment of the leishmaniasis. Although, according to the results of the previous studies, none of the developed vaccines has been approved as a definitive treatment for this disease $(20,21)$. However, among them, DNA vaccines have been shown to be more suitable due to the activation of major histocompatibility complex I (MHCl) and major histocompatibility complex II (MHCII) and induction of the appropriate response of Th1 and Tc in the host $(22,23)$.

Among the DNA vaccines evaluated in the treatment of various diseases, multi-epitope DNA vaccines designed by the immunoinformatic software in several stages in -silico have been reported to be successful in the in -vivo studies (24). In fact, peptides are better immunogens than the whole proteins, which can prevent the allergic reactions, cross-reactions, as well as the pathological reactions, because these epitopes are designed in -silico before experimental assay. Then, the gene sequence generating these multi-epitope DNA vaccines can be entered into the suitable vector after designing and it can be added to the host $(25,26)$.

Designed epitopes are exactly the same areas of the target immunogens that can activate the Tc and Th1 cells to stimulate the cellular immunity and B-cells to stimulate the humoral immunity. As a result, it can be expected that the designed multi-epitope vaccine can be a good candidate for vaccination (27).

Although, the short sequence of these vaccines can cause them to rapidly degrade within the cell, using viral vectors to transfer them into the cell can lead to the increased stability $(28,29)$. Nowadays, lentiviral vaccines have received a great deal of attention due to the rapid proliferation of the virus in the body and the lack of need for adjuvant because these vaccines can activate the cellular immunity by enhancing the Th1 and Tc responses $(30,31)$. These viral vectors can contain the desired protein or polypeptide for different types of vaccines.

In our previous study, immunogenicity evaluation of the whole protein of KMP11 and HASPB against $L$. major was successful in the BALB/c mice (14). Therefore, in this study, for the first time, the immunogenicity of the KMP11 and HASPB as selected epitopes of the $L$ - major was evaluated as a novel lentiviral multi-epitope vaccine. 
After designing the vaccine, $4 \mathrm{CTL}$ (or CD8+) and $2 \mathrm{HTL}$ (or CD4+) selected epitopes were linked together using the GDGDG linker. This linker is composed of the glycine (Gly) and aspartic acid (Asp), which are flexible and hydrophilic, respectively. This linker can facilitate the expression of the multi-epitope vaccine (32).

Then, the vaccine was subcloned in the PCDH513 lentiviral vector and the immunogenic effect of the vaccine was assessed through determination of interferon gamma (IFN- $\gamma$ ) and interleukin 4(IL-4) cytokines as well as titer of immunoglobulin G 2a (IgG2a) and immunoglobulin G 1 (IgG1) antibodies. In fact, the cytokines and antibodies are studied due to the process occurring after the parasite enters the host and during vaccination where the vaccine attempts to stimulate the defense against the parasite.

After the parasite enters the host, dendritic cells are activated, and the immune system changes with respect to Th1 response and IFN-y production increases by stimulating the interleukin 12(IL-12) production. Studies have shown that M1 macrophages are stimulated to produce the nitric oxide (NO) by the inducible nitric oxide synthase (iNOS) and kill the parasites by increasing the level of IFN-Y against the parasite $(33,34)$. Also, an increase in the IFN- $y$ level can lead to activation of Tc, natural killer (NK), and opsonin antibodies, such as IgG2a (35). On the other hand, increased level of cytokines, such as IL-4 and interleukin 23(IL-23) against immunological agents can lead to the altered immune responses to Th2 and IgG1 antibodies $(12,36)$.

In our study, the levels of IFN-y and IL-4 cytokines were significantly increased in the group immunized with the rLV-multi-epitope vaccine compared to the control groups $(p<0.05)$. Also, due to the importance of the balance between the levels of IFN- $\gamma$ and IL-4, the ratio of these two cytokines was measured and the results showed a significant increase in this ratio in the immunized group compared to the control group $(P<0.05)$. The increase in this ratio indicated that the splenocytes can induce the memory T-cells and cellular immunity was stimulated in the appropriate direction against the parasite, and Th1 and Tc were dominant to Th2 in the host. In addition, isotype switching occurs and IgG2a is produced with the increase in the IFN- $y$ level, which can stimulate the humoral immunity in defense against the parasites. However, researchers have not yet reached a definitive conclusion about the role of antibodies (37).

In the present study, the levels of $\lg$ G2a and IgG1 were assessed and according to the results, these antibodies were increased in the group vaccinated with the rLV-multi-epitope $(p<0.05)$. Moreover, the ratio of $\operatorname{lgG} 2$ a to $\lg G 1$ production in the immunized group was increased significantly compared to the control group $(P<0.05)$. Since, IgG2a production can indicate the progression of the cellular immune pathway to Th1 and IgG1 production can indicate the progression of the cellular immune pathway to Th2, it can be inferred that the Th1 is dominant to the Th2 and possibly it stimulates the host immune system to defend against the parasites.

Hua Cong et al., in a study on the vaccination against the Toxoplasma gondii by a multi- epitope DNA vaccine showed that the vaccine was capable of stimulating the cellular and humoral immunity (36). Also, Li et al., and Escalona et al., in their studies found that the cellular and humoral immunity was stimulated with the increase in the production of IFN- $y$ cytokine and IgG2a antibodies using the multi- 
epitope DNA vaccine against the Epstein-Barr virus (EBV) and Brucella Abortus and the vaccine was effective $(38,39)$. Also, Salehi et al., demonstrated that the use of a polyepitope DNA vaccine expressing the KMP11, Leishmania major stress-inducible protein1 (LmsTI1), thiol-specific antioxidant protein (TSA), and Leishmania homologue of receptors for activated $C$ kinase (LACK) against the leishmaniasis caused an increase in the level of IFN- $y$ and a Th1 response, although the load of parasite was decreased in the liver after the challenge (40). These studies are consistent with the results of our research, although more research is needed on the design and synthesis of these vaccines.

\section{Conclusion}

Results of the present study revealed that the developed recombinant lentiviral vaccine expressing the KMP11 and HASPB multi-epitope was able to create a protective effect in the BALB /c mice. Increased efficacy of the vaccine was confirmed as the initial design of the vaccine was performed in -silico. In addition, less time and cost is required for synthesis of these vaccines compared to the whole protein with the help of immunoinformatics server. Therefore, it is hoped that this vaccine will be economically viable. Our results showed that the rLV-multi-epitope vaccine could stimulate the immune system towards the Th1 well, stimulating the production of Tc and NK, as well as the formation of M1 macrophage by producing the protective cytokines including IFN- $\gamma$, consequently leading to the control of the disease. It also contributes to the eradication of the parasites by producing the opsonin antibodies including IgG2a. Although, the results showed that our vaccine can be prophylactic and therapeutic, more tests are needed including the challenge with the parasite, and more cytokine assessments for final confirmation.

\section{Abbreviations}

CL

Cutaneus Leishmaniasis

VL

visceral leishmaniasis

IFN-Y

interferony

IL-4

interleukin-4

IgG

immunoglobulin $\mathrm{G}$

KMP11

kinetoplastid membrane protein 11

HASPB

hydrophilic acylated surface protein B

LV

lenti virus 


\section{Declarations}

\section{Ethics approval and consent to participate}

The animal study was done based on the specific National Ethical Guidelines for Biomedical Research issued by the Research and Technology Deputy of Ministry of Health and Medicinal Education (MOHME) of Iran (2005).

\section{Consent for publication}

Not applicable.

\section{Availability of data and materials}

All data analysed during this study are included in this published article.

\section{Conflict of Interest}

The authors declare no competing interests

\section{Funding}

This study was supported by: Fasa University of medical sciences Grant No. 96175

\section{Author Contributions}

A.F, N.M, and Z.R conceived and designs the study and collected the data. M.R and N.M, A.F and A.M performed the experiments. A.Gh and A.A helped with cytokine analyses and cell culture. M.R, A.F wrote the first draft of the manuscript. All authors read and approved the final manuscript.

\section{Acknowledgements:}

The authors appreciate Fasa University of medical sciences for financial supports of this work.

\section{References}

1. Elikaee S, Mohebali M, Rezaei S, Eslami H, Khamesipour A, Keshavarz H, et al. Leishmania major p27 gene knockout as a novel live attenuated vaccine candidate: Protective immunity and efficacy evaluation against cutaneous and visceral leishmaniasis in BALB/c mice. Vaccine. 2019;37(24):3221-8.

2. Nguyen AK, Yang KH, Bryant K, Li J, Joice AC, Werbovetz KA, et al. Microneedle-Based Delivery of Amphotericin B for Treatment of Cutaneous Leishmaniasis. Biomed Microdevices. 2019;21(1):8.

3. Zutshi S, Kumar S, Chauhan P, Bansode Y, Nair A, Roy S, et al. Anti-Leishmanial Vaccines: Assumptions, Approaches, and Annulments. Vaccines (Basel). 2019;7(4). 
4. Osman M, Mistry A, Keding A, Gabe R, Cook E, Forrester S, et al. A third generation vaccine for human visceral leishmaniasis and post kala azar dermal leishmaniasis: First-in-human trial of ChAd63-KH. PLoS Negl Trop Dis. 2017;11(5):e0005527.

5. Bhavana Sethu Lakshmia RW. Rentala Madhubala. Leishmania genome analysis and highthroughput immunological screening identifies tuzin as a novel vaccine candidate against visceral leishmaniasis. Vaccine. 2014.

6. Solana JC, Ramirez L, Cook EC, Hernandez-Garcia E, Sacristan S, Martin ME, et al. Subcutaneous Immunization of Leishmania HSP70-II Null Mutant Line Reduces the Severity of the Experimental Visceral Leishmaniasis in BALB/c Mice. Vaccines (Basel). 2020;8(1).

7. Mirza MU, Rafique S, Ali A, Munir M, Ikram N, Manan A, et al. Towards peptide vaccines against Zika virus: Immunoinformatics combined with molecular dynamics simulations to predict antigenic epitopes of Zika viral proteins. Sci Rep. 2016;6:37313.

8. Ali M, Pandey RK, Khatoon N, Narula A, Mishra A, Prajapati VK. Exploring dengue genome to construct a multi-epitope based subunit vaccine by utilizing immunoinformatics approach to battle against dengue infection. Sci Rep. 2017;7(1):9232.

9. Pritam M, Singh G, Swaroop S, Singh AK, Pandey B, Singh SP. A cutting-edge immunoinformatics approach for design of multi-epitope oral vaccine against dreadful human malaria. Int J Biol Macromol. 2020;158:159-79.

10. Yang M, Zhu F, Sonderstrup G, Eckels DD. Recognition of endogenously synthesized HLA-DR4 restricted HCV epitopes presented by autologous EBV transformed B-lymphoblastoid cell line. Vaccine. 2005;23(7):951-62.

11. Ikram A, Zaheer T, Awan FM, Obaid A, Naz A, Hanif R, et al. Exploring NS3/4A, NS5A and NS5B proteins to design conserved subunit multi-epitope vaccine against $\mathrm{HCV}$ utilizing immunoinformatics approaches. Sci Rep. 2018;8(1):16107.

12. Thema N, Tshilwane SI, Pretorius A, Son L, Smith RM, Steyn HC, et al. Identification and characterisation of conserved epitopes of E. ruminantium that activate Th1 CD4(+) T cells: Towards the development of a multi-epitope vaccine. Mol Immunol. 2019;107:106-14.

13. van Rosmalen $M$, Krom M, Merkx M. Tuning the Flexibility of Glycine-Serine Linkers To Allow Rational Design of Multidomain Proteins. Biochemistry. 2017;56(50):6565-74.

14. Mortazavidehkordi N, Fallah A, Abdollahi A, Kia V, Khanahmad H, Najafabadi ZG, et al. A lentiviral vaccine expressing KMP11-HASPB fusion protein increases immune response to Leishmania major in BALB/C. Parasitol Res. 2018;117(7):2265-73.

15. Rabienia M, Roudbari Z, Ghanbariasad A, Abdollahi A, Mohammadi E, Mortazavidehkordi N, Farjadfar A. Exploring membrane proteins of Leishmania major to design a new multi-epitope vaccine using immunoinformatics approach. Eur J Pharm Sci. 2020 Sep 1;152:105423.

16. Barde I, Salmon P, Trono D. Production and titration of lentiviral vectors. Curr Protoc Neurosci. 2010;Chap. 4:Unit 421. 
17. Kutner RH, Zhang XY, Reiser J. Production, concentration and titration of pseudotyped HIV-1-based lentiviral vectors. Nat Protoc. 2009;4(4):495-505.

18. Shen WF, Galula JU, Liu JH, Liao MY, Huang CH, Wang YC, et al. Epitope resurfacing on dengue viruslike particle vaccine preparation to induce broad neutralizing antibody. Elife. 2018;7.

19. Mortazavi Dehkordi N, Ghaffarifar F, Mohammad Hassan Z, Esavand Heydari F. In Vitro and In Vivo Studies of Antileishmanial Effect of Artemether on Leishmania infantum. Jundishapur Journal of Microbiology. 2013;6(5).

20. Palatnik-de-Sousa CB, Nico D. The Delay in the Licensing of Protozoal Vaccines: A Comparative History. Front Immunol. 2020;11:204.

21. Misra P, Tandon R, Basak T, Sengupta S, Dube A. Purified Splenic amastigotes of Leishmania donovani-Immunoproteomic approach for exploring Th1 stimulatory polyproteins. Parasite Immunol. 2020:e12729.

22. Madhubala BSaR. Leishmaniasis: Current Status of Vaccine Development. Current Molecular Medicine. 2004.

23. Bazhan SI, Antonets DV, Karpenko LI, Oreshkova SF, Kaplina ON, Starostina EV, et al. In silico Designed Ebola Virus T-Cell Multi-Epitope DNA Vaccine Constructions Are Immunogenic in Mice. Vaccines (Basel). 2019;7(2).

24. Zhang X, Liu S, Li S, Du Y, Dou Y, Li Z, et al. Designation of a novel DKK1 multiepitope DNA vaccine and inhibition of bone loss in collagen-induced arthritic mice. Biomed Res Int. 2015;765490.

25. Purcell AW, McCluskey J, Rossjohn J. More than one reason to rethink the use of peptides in vaccine design. Nat Rev Drug Discov. 2007;6(5):404-14.

26. Aguilar JC, Rodriguez EG. Vaccine adjuvants revisited. Vaccine. 2007;25(19):3752-62.

27. Tshilwane SI, Thema N, Steyn HC, van Kleef M, Pretorius A. A multi-epitope DNA vaccine coadministered with monophosphoryl lipid A adjuvant provides protection against tick transmitted Ehrlichia ruminantium in sheep. Vaccine. 2019;37(31):4354-63.

28. De Brito RCF, Cardoso JMO, Reis LES, Vieira JF, Mathias FAS, Roatt BM, et al. Peptide Vaccines for Leishmaniasis. Front Immunol. 2018;9:1043.

29. Ewer KJ, Lambe T, Rollier CS, Spencer AJ, Hill AV, Dorrell L. Viral vectors as vaccine platforms: from immunogenicity to impact. Curr Opin Immunol. 2016;41:47-54.

30. Cartier N, Hacein-Bey-Abina S, Bartholomae CC, Bougneres P, Schmidt M, Kalle CV, et al. Lentiviral hematopoietic cell gene therapy for X-linked adrenoleukodystrophy. Methods Enzymol. 2012;507:187-98.

31. Robyn AA, Oldham M, Elliot M, Berinstein JA. Lentiviral vectors in cancer immunotherapy. Immunotherapy. 2015.

32. Brian Livingston CC, Newman M, Higashimoto Y, Appella E, Sidney J, Alessandro Sette. A Rational Strategy to Design Multiepitope Immunogens Based on Multiple Th Lymphocyte Epitopes. The Journal of Immunology. 2002. 
33. Reza S, Hasan NA, Maryam NF, Fahimeh B, Ghahremani A, GholamReza H, et al. Cytokine profile and nitric oxide levels in macrophages exposed to Leishmania infantum FML. Exp Parasitol. 2019;203:1-7.

34. Bhattacharya P, Dey R, Dagur PK, Kruhlak M, Ismail N, Debrabant A, et al. Genetically Modified Live Attenuated Leishmania donovani Parasites Induce Innate Immunity through Classical Activation of Macrophages That Direct the Th1 Response in Mice. Infect Immun. 2015;83(10):3800-15.

35. Mohr E, Cunningham AF, Toellner KM, Bobat S, Coughlan RE, Bird RA, et al. IFN-\{gamma\} produced by CD8 T cells induces T-bet-dependent and -independent class switching in B cells in responses to alum-precipitated protein vaccine. Proc Natl Acad Sci U S A. 2010;107(40):17292-7.

36. Cong H, Gu QM, Yin HE, Wang JW, Zhao QL, Zhou HY, et al. Multi-epitope DNA vaccine linked to the A2/B subunit of cholera toxin protect mice against Toxoplasma gondii. Vaccine. 2008;26(31):391321.

37. Miles SA, Conrad SM, Alves RG, Jeronimo SM, Mosser DM. A role for IgG immune complexes during infection with the intracellular pathogen Leishmania. J Exp Med. 2005;201(5):747-54.

38. Escalona E, Saez D, Onate A. Immunogenicity of a Multi-Epitope DNA Vaccine Encoding Epitopes from Cu-Zn Superoxide Dismutase and Open Reading Frames of Brucella abortus in Mice. Front Immunol. 2017;8:125.

39. Li W, Chen Q, Chen H, Rao P, Xue X, Chen S, et al. Immune response of mice to a latency membrane protein 2 multiepitope antigen of Epstein-Barr virus applied as DNA vaccine and/or peptide vaccine. Acta Virol. 2013;57(1):51-8.

40. Salehi-Sangani G, Mohebali M, Jajarmi V, Khamesipour A, Bandehpour M, Mahmoudi M, et al. Immunization against Leishmania major infection in BALB/c mice using a subunit-based DNA vaccine derived from TSA, LmSTI1, KMP11, and LACK predominant antigens. Iran J Basic Med Sci. 2019;22(12):1493-501.

\section{Figures}




\section{GDGDG Linker}

A

\section{Muliti-epitope vaccine}
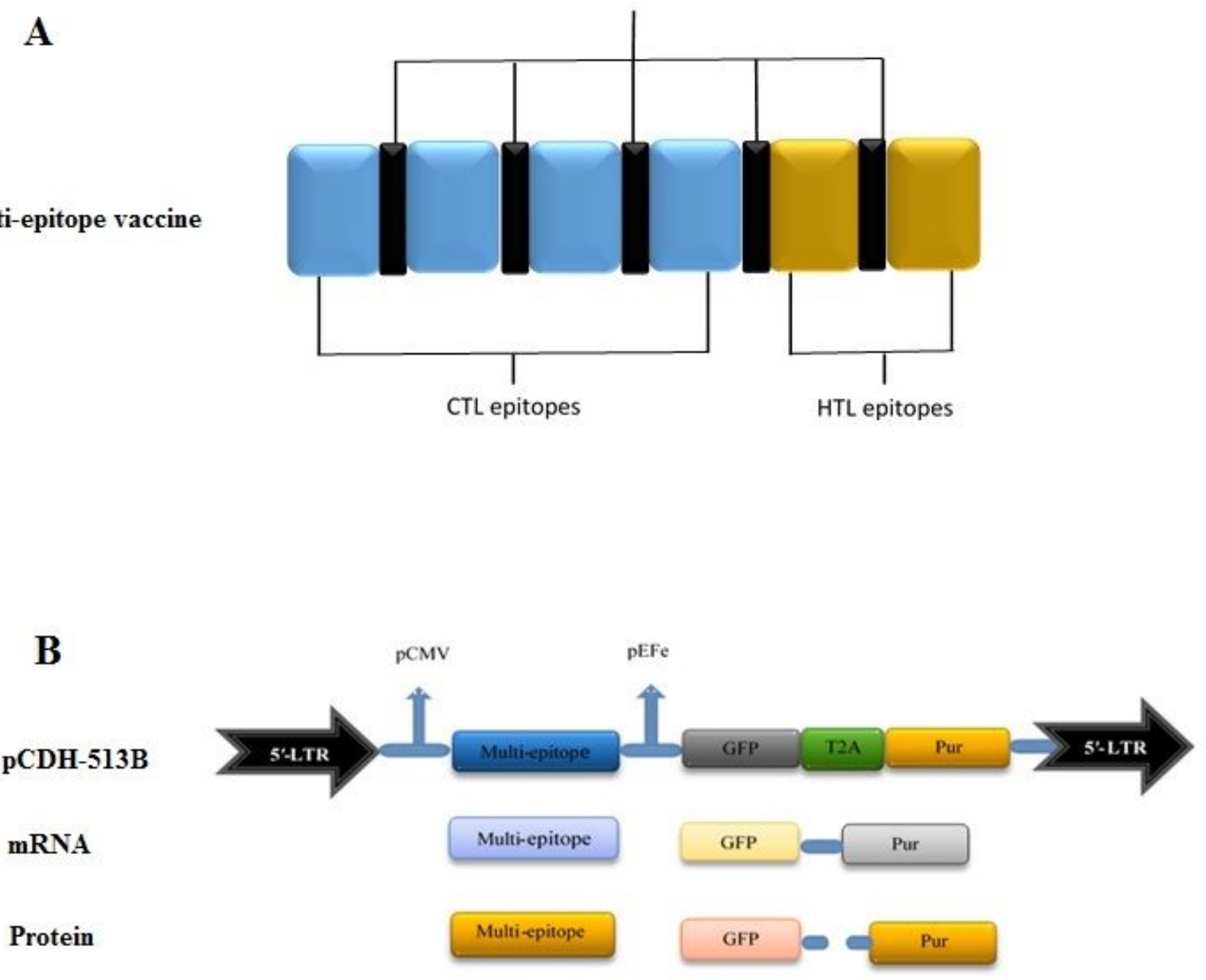

Figure 1

Schematic diagram of final designed vaccine. Selected epitopes in silico were joined together by GDGDG linker (blank). The light blue rectangles are representing CTL epitopes and light brown rectangle also showing the HTL epitopes. 

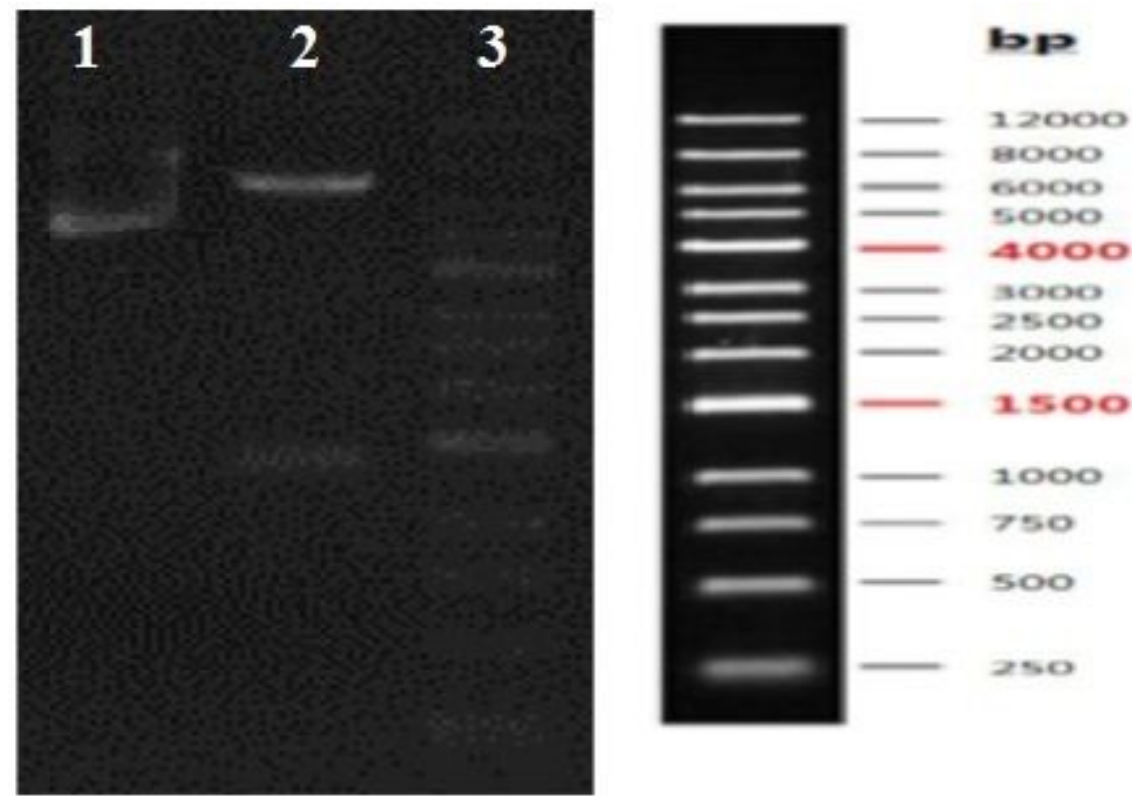

Figure 2

Schematic illustration of pCDH-Multiepitope vaccine. The multi-epitope sequence was synthesized by Genscript, USA, and were incorporated into pCDH plasmid. In this construct, using cytomegalovirus (CMV) and eukaryotic elongation factor (eEF) promoters, the multi-epitope, GFP and puromycin were transcribed, respectively. Finally, transfer vector expresses multi-epitope, GFP and puromycin resistance factor from two mRNAs.
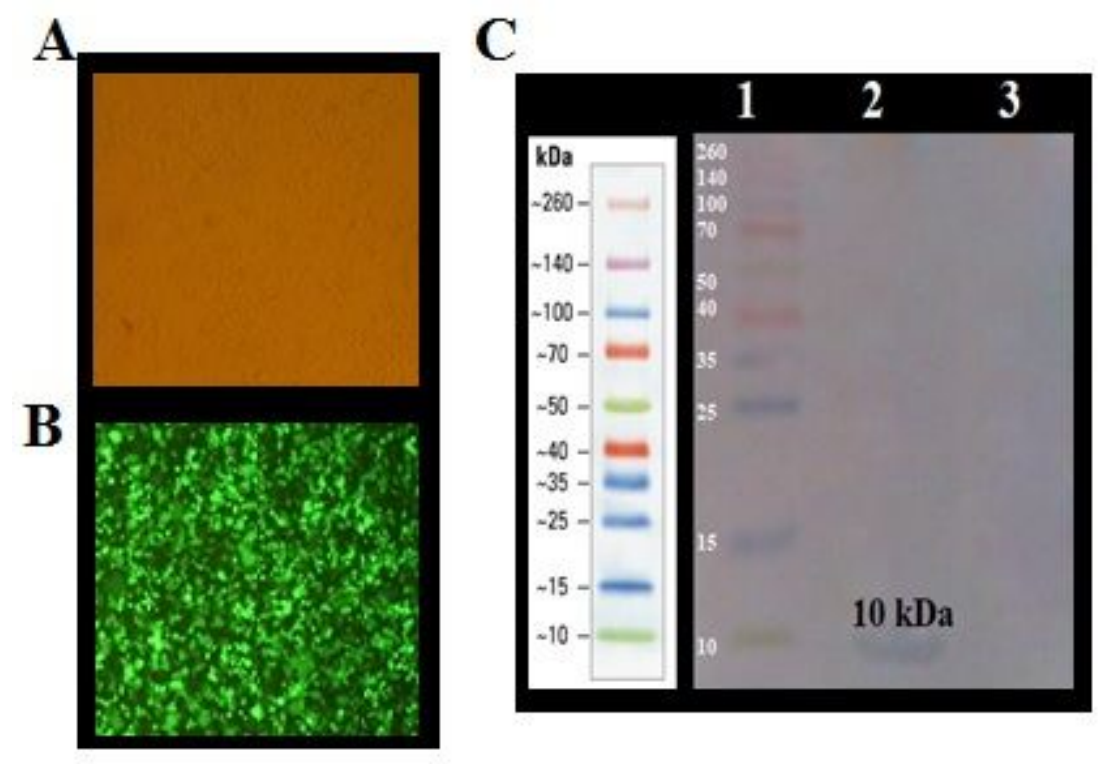

Figure 3

Construction of recombinant lentiviral vector. Using Xbal and Apal restriction enzymes, one fragment with length of 1478 and $6355 \mathrm{bp}$ were attained from analysis of rLV- multi-epitope. Lane 1: undigested plasmid Lane 2: digested plasmid Lane 3: 1Kb DNA marker 
A

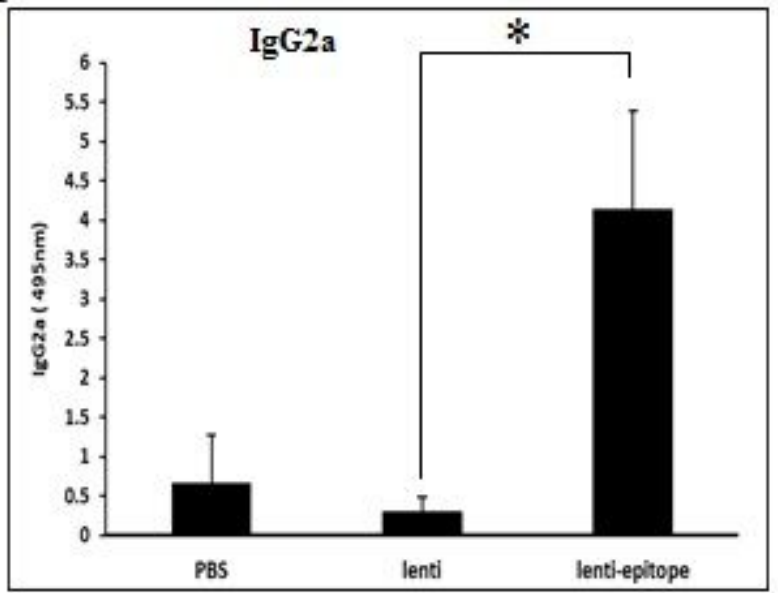

B

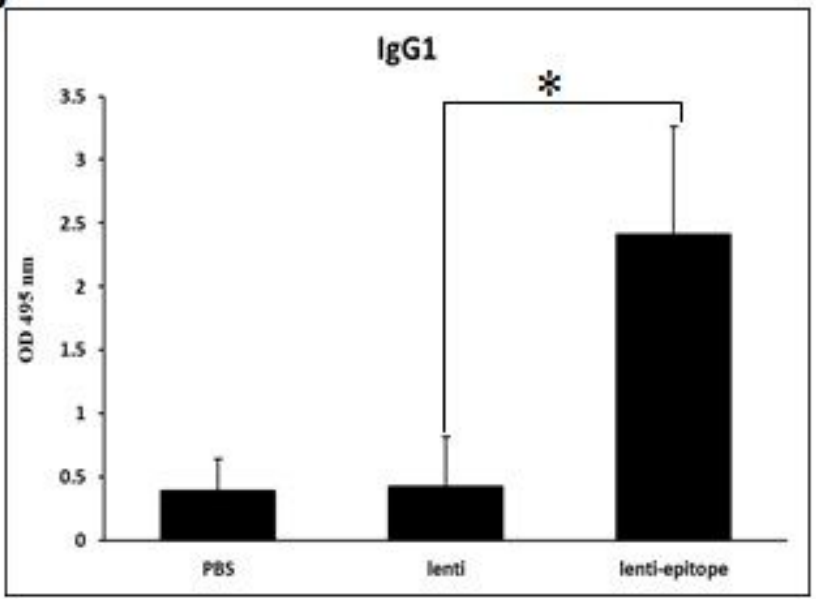

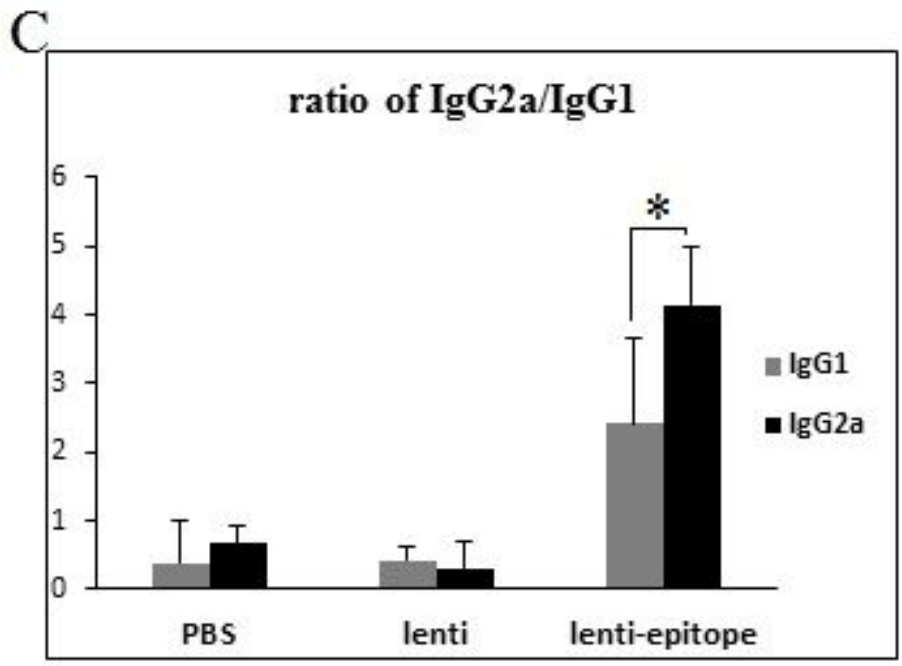

Figure 4

Transfection of HEK293T using three plasmids (pCDH, psPAX, and pMD2), transduction of HEK293T using lentiviral particles and Western blot. (a) HEK293T before transfection (b) HEK293T cells 72h after transduction (down) using rLV-Multiepitope showed more than $90 \%$ of the cells were successfully transducted. (C) Immunoblotting was done using Anti-His antibody conjugated with horseradish peroxidase against Histag (Abcam,USA). A $10 \mathrm{KD}$ band of multi-epitope confirmed the presence of the multi-epitope in the HEK293T cell lysate of rLV-Multiepitope. Also, there was no band detected in control cells (transduced with the rLV-empty) 

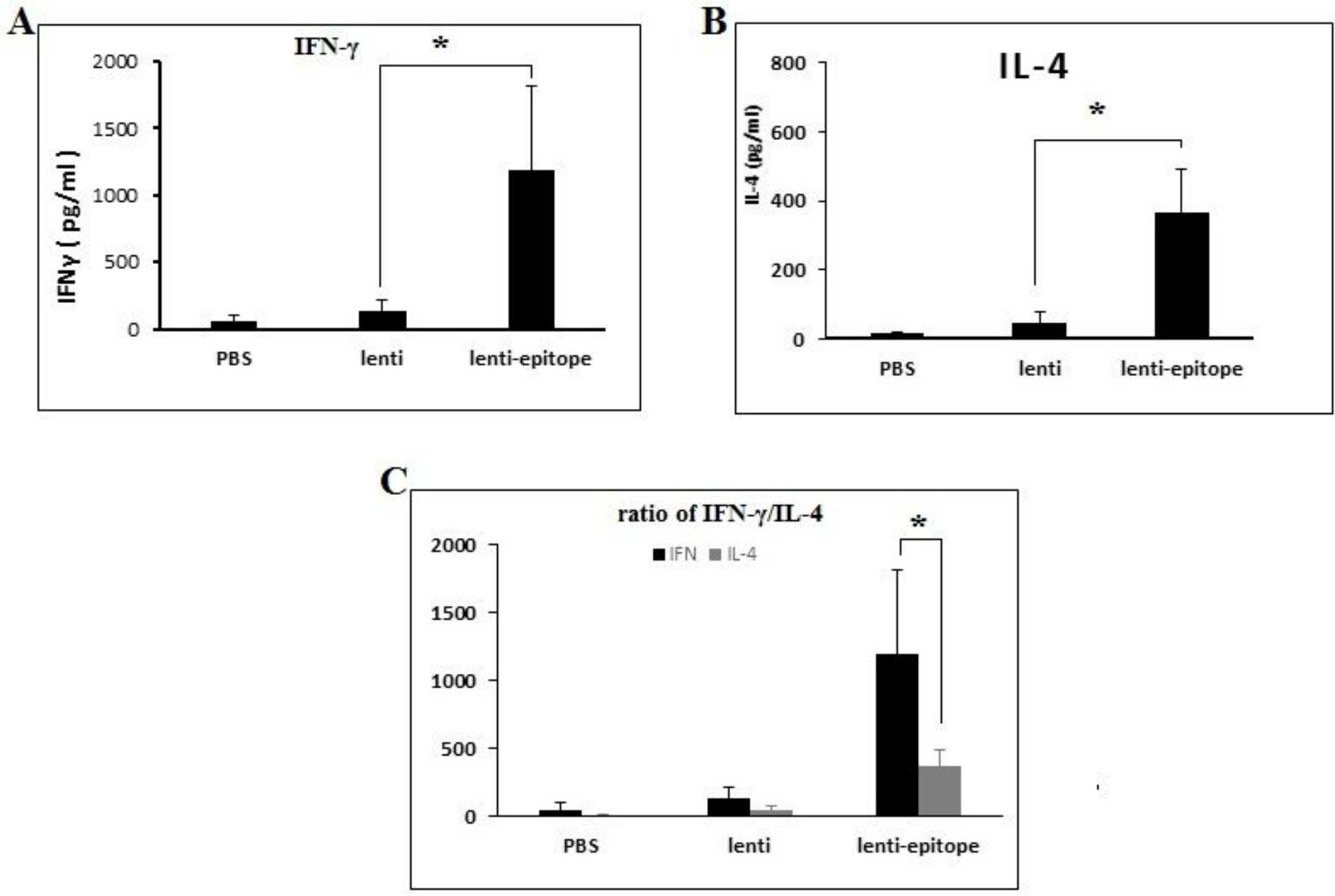

Figure 5

Analysis of IFN-y and IL-4 levels in the supernatants of splenocytes stimulated with lysate of L. major. (A) The consentration of IFN- $\gamma$ produced by splenocytes of rLV-Multi-epitope vaccinated mice was significantly higher than that of two control groups (rLV-empty and PBS). (B) IL-4 produced by splenocytes of rLV-multi-epitope vaccinated mice was significantly higher than that of two control groups (rLV-empty and PBS). Moreover, the level of IFN-y was significantly higher than that of IL-4 (C). Data are presented as means $\pm S D$. All tests were done in triplicate, ${ }^{*} P<0.05$

\section{Image not available with this version}




\section{Figure 6}

The level of multi-epitope IgG antibody in the sera of immunized mice. Accoding to ELISA test, three weeks after injection, the titer of $\lg G 2 a(A), \lg G 1$ (B) antibodies against L. major in the sera of the mice immunized with rLV-Multi-epitope were detected. The consentration of IgG1 and IgG2a produced by rLVMulti-epitope immunized mice were significantly higher than that of two control groups (rLV-empty and PBS). The results showed that the immunized mice had a significant increase in ratio of IgG2a in comparison to $\lg \mathrm{G} 1(\mathrm{C})$. Data were presented as means $\pm S D$. All tests were done in triplicate. ${ }^{*} \mathrm{P}<0.05$

\section{Supplementary Files}

This is a list of supplementary files associated with this preprint. Click to download.

- graficalabstract.png 